\title{
Low Risk of Recurrence After Posterior Labral Repair of the Shoulder in a High-Risk United States Military Population
}

\author{
Stephen Chan, M.D., Lisa K. O’Brien, D.O., Brian R. Waterman, M.D., \\ Andrew G. Chan, M.D., M.P.H., Mark Pallis, D.O., and Kelly G. Kilcoyne, M.D.
}

\begin{abstract}
Purpose: To examine the results of isolated arthroscopic posterior labral repair of the shoulder in an active military population, looking specifically at the reoperation rate and rate of return to previous military activity with a minimum follow-up period of 2 years. Methods: A retrospective case series was performed in active-duty military service members who underwent isolated, primary arthroscopic posterior labral repair at a single academic military treatment facility between 2009 and 2015 and had at least 2 years of follow-up. Patients were excluded if they were of non-active-duty status, had insufficient follow-up ( $<2$ years), or had undergone a concurrent procedure. Injury presentation, demographic data, and surgical data (i.e., surgical positioning, number of anchors, and anchor placement location) were compiled manually. Outcomes including the rate of return to active duty, recurrence of symptoms, and need for revision surgery were evaluated. Results: Sixty-five patients were included. After arthroscopic repair, a high rate of return to previous military duties $(83 \%)$ was noted at short- to mid-term follow-up (mean \pm standard deviation, $3.04 \pm 1.30$ years), with 1 patient $(1.5 \%)$ requiring revision arthroscopic repair and 10 patients $(15.5 \%)$ showing activity-limiting shoulder pain preventing a return to active duty. Intraoperative positioning $(P=.17)$, a low anchor position $(P=.27)$, and the number of anchors used $(P=.62)$ were not found to be significant contributors to continued postoperative pain or recurrent instability. Conclusions: Arthroscopic intervention resulted in a reliable rate of glenohumeral stability with a low rate of surgical revision and a high rate of return to military duty at short- to mid-term follow-up. However, 1 in 6 military service members showed significant, activity-limiting shoulder pain postoperatively that did not permit a return to previous military activities after surgical intervention. Level of Evidence: Level IV, therapeutic case series.
\end{abstract}

$\mathbf{P}$ osterior glenohumeral instability is relatively uncommon within the literature, representing $2 \%$ to $10 \%$ of all cases of shoulder instability. ${ }^{1,2}$ Posterior instability comprises a spectrum of pathology that can result from chronic microtrauma, from recurrent

From the Department of Orthopaedic Surgery, William Beaumont Army Medical Center (S.C., A.G.C., M.P., K.G.K.), El Paso, Texas; and Department of Orthopaedic Surgery, Wake Forest Baptist Medical Center (L.K.O., B.R.W.), Winston-Salem, North Carolina, U.S.A.

The authors report that they have no conflicts of interest in the authorship and publication of this article. Full ICMJE author disclosure forms are available for this article online, as supplementary material.

Received June 22, 2019; accepted October 27, 2019.

Address correspondence to Kelly G. Kilcoyne, M.D., William Beaumont Army Medical Center, 5005 N Piedras St, El Paso, TX 79920, U.S.A. E-mail: kelly.g.kilcoyne.mil@mail.mil

Published by Elsevier on behalf of the Arthroscopy Association of North America. This is an open access article under the CC BY-NC-ND license (http://creativecommons.org/licenses/by-nc-nd/4.0/).

2666-061X/19778

https://doi.org/10.1016/j.asmr.2019.10.008 posterior subluxations, or most rarely, from a frank dislocation, and it can involve the posterior labrum, capsule, glenohumeral ligaments, or bony articulation. ${ }^{3-11}$ The pathophysiology of these injuries is complex, and its presentation can be highly variable, with the most common presentation being subtle symptoms of pain or weakness without a known traumatic event. ${ }^{1-4}$

Despite its scarcity, posterior instability has been increasingly recognized as a source of shoulder disability in the athletic population. ${ }^{1-8}$ The mechanism of injury most classically described is repetitive eccentric posterior glenoid edge loading, such as occurs in defensive linemen or patients who regularly engage in exercises such as push-ups and bench press. Other athletes who are at risk of posterior labral injury are overhead athletes such as baseball players and swimmers. ${ }^{8}$

Although not prominent in the literature, military personnel are likely also at increased risk of posterior labral tears owing to the extraordinary demands 
required of them. An epidemiologic study by Owens et al. ${ }^{11}$ identified $10.3 \%$ of overall shoulder instability to be posterior in the military population. Military personnel are required to routinely lift and carry heavy weights overhead, climb, crawl, and negotiate obstacles or difficult terrain and to engage in daily upper-body strengthening (e.g., push-ups) and conditioning necessary to maintain the requisite physical fitness for military service.

The trend in managing posterior labral injuries has shifted toward early arthroscopic stabilization after a brief trial of conservative treatment. ${ }^{1,4,10,12}$ A systematic review and meta-analysis by DeLong et al. ${ }^{12}$ found arthroscopic posterior labral repair to be effective and reliable in 27 studies involving contact and overhead athletes, with a high rate of return to sport $(92 \%)$. However, few studies have investigated the results of arthroscopic treatment of posterior shoulder instability in an active-duty military population, including the rate of return to previous military activity and revision rate. ${ }^{3,11}$ Isolated posterior labral repairs constitute up to $24 \%$ of operatively treated labral repairs in a military population, which is higher than reported in previous studies. ${ }^{3,11-14}$

The purpose of this study was to examine the results of isolated arthroscopic posterior labral repair of the shoulder in an active military population, looking specifically at the reoperation rate and rate of return to previous military activity with a minimum follow-up period of 2 years. We hypothesized that most service members would capably return to duty with a low rate of revision surgery.

\section{Methods}

A retrospective case series was performed to assess all active-duty military service members who underwent isolated, primary arthroscopic posterior labral repair at a single academic military treatment facility by 7 surgeons (with a range of 5-7 years of experience) between 2009 and 2015 and had at least 2 years of followup. Operative reports and patient charts were obtained using Essentris (version 213.02; CliniComp International, San Diego, CA) (Department of Defense Inpatient Electronic Health Record System) and the Armed Forces Health Longitudinal Technology Application (AHLTA, version 3.3; Defense Health Information Management System, Falls Church, VA). Patients who underwent a shoulder labral repair were searched for and identified by querying the Department of Defense Military Surgical Scheduling System (S3, version 2.3). A thorough search through Essentris and AHLTA confirmed that the labral repairs were of the posterior labrum.

Patients were included in our study if they were of active-duty status; underwent an isolated, primary posterior labral repair; and had at least 2 years of postoperative follow-up. Patients were excluded if they were of non-active-duty status at the time of surgery; had insufficient follow-up ( $<2$ years); or had undergone a concurrent procedure, such as an adjacent (anterior or superior) labral repair or rotator cuff repair. All identified cases of isolated arthroscopic posterior labral repair that met the inclusion criteria were then compiled for further analysis. The electronic medical records and imaging software were reviewed to extract pertinent patient demographic characteristics, mechanisms of injury, physical examination findings, imaging, surgical technique, and perioperative clinical course (from time of injury to final follow-up). Isolated posterior labral tears were confirmed with magnetic resonance arthrogram imaging by the treating surgeon. The indications for surgery were continued pain and/or instability after failure of a trial of conservative management, which consisted of activity modification, antiinflammatory medication, and physical therapy. The patient's medical record, demographic variables (i.e., age, sex, rank, and military occupational specialty), laterality, injury characteristics (i.e., presence of traumatic event), and surgical history were extracted. Mechanisms of injury were stratified into either (1) direct trauma, (2) insidious onset, or (3) seizure event. Variables from preoperative physical examination maneuvers that were documented were the Kim test, ${ }^{15}$ posterior load-and-shift test, ${ }^{16}$ and jerk test. ${ }^{16}$ In addition, the clinical course was analyzed to determine surgical variables (i.e., perioperative complications, surgical technique, and revision surgery) and occupational outcomes (i.e., medical discharge, return to military duty, and permanent activity limitations). Active-duty disposition after surgery was determined based on US Army Physical Disability Agency or AHLTA notation. The primary outcome was identified as surgical failure due to recurrent instability and requiring revision posterior stabilization. Secondary outcomes were defined as clinical failures, which included the inability to return to preoperative military duties because of persistent shoulder pain or dysfunction, resulting in medical separation from the military. In addition, the rate of return to previous military duty was determined, and selected variables (age, sex, arm dominance, injury characteristics, preoperative physical examination, surgical positioning, number of anchors used, and placement location of anchors) were evaluated to determine the risk factors and odds ratios (ORs) for persistent postoperative pain and recurrent posterior instability.

\section{Surgical Technique}

All arthroscopic posterior labral repairs were performed at a single institution by 1 of 7 attending orthopaedic surgeons. Patient position, anchor type, and number were at the discretion of the treating surgeon (Table 1). A preoperative examination with the patient 
Table 1. Summary of Demographic, Preoperative Clinical, and Surgical Characteristics $(\mathrm{N}=65)$

\begin{tabular}{|c|c|}
\hline Risk Factor & Data \\
\hline$\overline{\text { Age (continuous), mean } \pm S D \text {, yr }}$ & $27.8 \pm 6.6(\mathrm{~N}=65)$ \\
\hline \multicolumn{2}{|l|}{ Sex } \\
\hline Female & $5(7.7)$ \\
\hline Male & $60(92.3)$ \\
\hline \multicolumn{2}{|l|}{ Dominant arm } \\
\hline Yes & $37(56.9)$ \\
\hline No & $28(43.1)$ \\
\hline \multicolumn{2}{|l|}{ Mechanism of injury } \\
\hline Trauma or dislocation event & $43(66.2)$ \\
\hline Insidious onset & $21(32.3)$ \\
\hline Seizure event & $1(1.5)$ \\
\hline \multicolumn{2}{|l|}{ Preoperative chief complaint } \\
\hline Pain alone & $26(40.0)$ \\
\hline Instability & $6(9.2)$ \\
\hline Combined & $33(50.8)$ \\
\hline \multicolumn{2}{|l|}{ Physical examination maneuver } \\
\hline Kim test (preoperative) & $51(91)$ \\
\hline Jerk test (preoperative) & $60(95)$ \\
\hline \multicolumn{2}{|c|}{ Beach chair or lateral decubitus position } \\
\hline Beach chair & $15(23.1)$ \\
\hline Lateral decubitus & $50(76.9)$ \\
\hline \multicolumn{2}{|l|}{ No. of anchors } \\
\hline Mean \pm SD & $3.3 \pm 0.9(\mathrm{n}=63)$ \\
\hline$>3$ anchors & $21(33.3)$ \\
\hline$\leq 3$ anchors & $42(66.7)$ \\
\hline \multicolumn{2}{|l|}{ Anchor position } \\
\hline Low anchor present* & $41(85.4)$ \\
\hline No low anchor present & $7(14.6)$ \\
\hline
\end{tabular}

NOTE. Data are presented as frequency (percentage) unless otherwise indicated.

SD, standard deviation.

*Low anchor position defined as placement between 5- and 7o'clock positions.

under anesthesia was performed with comparison to the contralateral shoulder prior to positioning. The procedure was performed with general anesthesia with the patient in either the beach-chair or lateral decubitus position. A standard posterior portal was established first, followed by an anterior midglenoid portal within the rotator cuff interval. Diagnostic shoulder arthroscopy was then performed to evaluate the capsule, labrum, biceps tendon, rotator interval, axillary recess, rotator cuff, and articular surfaces of the glenoid and humeral head. After this, an anterior-superior portal was created, which was predominantly used for visualization with the arthroscope. The anterior midglenoid portal and posterior portal were used for instrumentation. On conclusion of the diagnostic portion of the procedure, a liberator was used to free up and mobilize the injured labrum. The medial neck of the posterior glenoid was further prepared to a bleeding surface using either a rasp or an arthroscopic shaver. Anchors were placed via the posterior portal or with an accessory percutaneous posterior-inferior (i.e., 7-o'clock) portal. The location and number of suture anchors used were dependent on the location and size of the labral tear, as well as surgeon preference. All surgeons routinely used 3.0-mm Bio-SutureTak or 2.4-mm BioComposite SutureTak suture anchors (Arthrex, Naples, FL). Suture passage was performed using an angled shuttling device (Suture Lasso; Arthrex) with superior advancement of the capsulolabral tissue using a "pinchtuck" configuration. Sutures in a simple suture configuration with single-loaded anchors were tied using standard arthroscopic knot tying with a sliding-knot configuration and reverse alternating half-hitches. Capsulolabral tissue was reapproximated to the inferior glenoid neck to reconstitute the posterior-inferior bumper, tension the posterior band of the inferior glenohumeral ligament, and re-center the humeral head on the glenoid. These steps were repeated for each anchor used in the repair and capsular plication.

Immediately after the procedure, patients were kept in a shoulder sling with an abduction pillow in neutral rotation for 6 weeks. During the recovery period, patients were not permitted to perform impact activities and were restricted from push-up-type movements. Passive range of motion was initiated at 2 weeks postoperatively, with progression to active range-of-motion exercise at 6 weeks and shoulder strengthening exercises at 12 weeks. After undergoing a functional assessment, patients were released to full participation in all activities at 6 months postoperatively, including contact sports, drills, and the Army Physical Fitness Test.

\section{Statistical Analysis}

Preoperative clinical and demographic characteristics were assessed with measures of central tendency. Statistical significance was assessed using $\chi^{2}$ and Fisher exact tests. $P<.05$ was deemed significant. Univariate and multivariate regression analyses were used to test our hypothesis with ORs and 95\% confidence intervals (CIs) adjusted for identified potential risk factors (summarized in Table 2) associated with poor outcomes and defined rates of failure. All risk factors were included in the multivariate models simultaneously.

\section{Results}

A total of 65 patients ( 60 men and 5 women) with a mean age of $27.8 \pm 6.6$ years were included in this study (Table 1). Of these patients, $61(94 \%)$ were of enlisted military rank and 37 (57\%) had involvement of the dominant extremity. All patients included in the study had no evidence of glenoid or humeral head bone loss based on record review. On quantification of primary injury mechanisms, 43 patients $(66 \%)$ sustained a single traumatic event, 21 patients $(32 \%)$ described an insidious onset of symptoms, and 1 patient $(2 \%)$ had a witnessed seizure. Patients commonly presented with a chief complaint of both pain and instability $(\mathrm{n}=33$, $51 \%)$, followed by pain only $(n=26,40 \%)$ and 
Table 2. Univariate Analysis of Risk Factors for Posterior Labral Tears of Shoulder in Active Military Population

\begin{tabular}{|c|c|c|c|}
\hline \multirow[b]{2}{*}{ Characteristic } & \multicolumn{3}{|c|}{ Clinical Failure } \\
\hline & OR & $\begin{array}{l}95 \% \mathrm{CI} \\
\text { for OR }\end{array}$ & $P$ Value \\
\hline Age (continuous) & 0.69 & $0.17-2.77$ & .60 \\
\hline \multicolumn{4}{|l|}{ Sex } \\
\hline Female vs male & 1.45 & $0.36-5.82$ & .60 \\
\hline Male vs female & 0.49 & $0.05-5.27$ & .56 \\
\hline Dominant arm & 0.85 & $0.09-8.27$ & .89 \\
\hline \multicolumn{4}{|l|}{ Chief complaint } \\
\hline $\begin{array}{l}\text { Pain alone vs } \\
\text { combined }\end{array}$ & 1.33 & $0.35-5.01$ & .68 \\
\hline Instability vs combined & 0.4 & $0.02-10.20$ & .58 \\
\hline $\begin{array}{l}\text { Pain alone vs } \\
\text { (instability or combined) }\end{array}$ & 1.62 & $0.42-6.27$ & .49 \\
\hline $\begin{array}{l}\text { (Pain alone }+ \text { instability) } \\
\text { vs combined }\end{array}$ & 1.04 & $0.27-3.99$ & .96 \\
\hline $\begin{array}{l}\text { (Pain alone }+ \text { combined }) \\
\text { vs instability }\end{array}$ & 2.76 & $0.12-66.3$ & .53 \\
\hline \multicolumn{4}{|l|}{ Mechanism of injury } \\
\hline $\begin{array}{l}\text { (Trauma or dislocation event) } \\
\text { vs insidious onset }\end{array}$ & 0.72 & $0.19-2.77$ & .63 \\
\hline $\begin{array}{l}\text { Insidious onset vs } \\
\text { (trauma or dislocation event) }\end{array}$ & 1.32 & $0.58-3.00$ & .50 \\
\hline Kim test & 0.53 & $0.10-2.79$ & .45 \\
\hline Jerk test & 1.9 & $0.36-10.07$ & .45 \\
\hline Beach chair vs lateral decubitus & 0.12 & $0.01-2.47$ & .17 \\
\hline No. of anchors & 0.44 & $0.02-11.18$ & .62 \\
\hline$>3$ anchors vs $\leq 3$ anchors & 2.29 & $0.09-58.54$ & .62 \\
\hline $\begin{array}{l}\text { Anchor position: low anchor } \\
\text { present vs no low } \\
\text { anchor present }\end{array}$ & 0.35 & $0.05-2.29$ & .27 \\
\hline
\end{tabular}

CI, confidence interval; OR, odds ratio.

symptomatic instability in isolation $(\mathrm{n}=6,9 \%)$ (Table 3). Provocative preoperative physical examination revealed confirmatory findings on the posterior load-and-shift test $(\mathrm{n}=64$; sensitivity, $98 \%$ ), jerk test ( $\mathrm{n}=60$; sensitivity, $95 \%)$, and Kim test $(\mathrm{n}=56$; sensitivity, $91 \%$ ).

At a mean of 3.04 years (standard deviation, 1.30 years) of postoperative follow-up, $83 \%$ of patients $(n=54)$ returned to their previous activities of military duty. Despite maintaining compliance with physical therapy, 1 patient $(1.5 \%)$ had persistent posterior shoulder instability. He ultimately required revision

Table 3. Outcomes After Posterior Labral Repair of Shoulder Based on Preoperative Chief Complaint

\begin{tabular}{lccc}
\hline \multicolumn{1}{c}{ Chief Complaint } & Total, $\mathrm{n}$ & $\begin{array}{c}\text { Clinical } \\
\text { Failure, }{ }^{*} \mathrm{n}(\%)\end{array}$ & $\begin{array}{c}\text { No Clinical } \\
\text { Failure, } \mathrm{n}(\%)\end{array}$ \\
\hline Pain alone & 26 & $5(19.2)$ & $21(80.8)$ \\
Instability alone & 6 & $0(0)$ & $6(100)$ \\
Combined $^{\dagger}$ & 33 & $5(15.2)$ & $28(84.8)$ \\
Pain alone + combined & 59 & $10(16.9)$ & $49(83.1)$ \\
\hline
\end{tabular}

*Defined as inability to return to preoperative military duties because of persistent shoulder pain or dysfunction, resulting in medical separation from military.

${ }^{\dagger}$ Defined as initial patient presentation of both pain and instability.
Table 4. Summary of Rate of Surgical and Clinical Outcomes After Posterior Labral Repair of Shoulder in Active Military Population

\begin{tabular}{lc}
\hline \multicolumn{1}{c}{ Measure } & Frequency $(\%)$ \\
\hline Clinical failure $^{*}$ & $10(15.5)$ \\
Surgical failure & \\
Overall failure & $1(1.5)$ \\
\hline
\end{tabular}

NOTE. Surgical failure is defined as recurrent instability that requires revision posterior stabilization. Clinical failure is defined as the inability to return to preoperative military duties because of persistent shoulder pain or dysfunction, resulting in medical separation from the military.

*Persistent activity-limiting shoulder pain, with failure to return to previous military activity.

${ }^{\dagger}$ Revision posterior labral repair.

surgery approximately 7 months after the index operation. He continued to have no improvement after revision surgery, resulting in separation from the military. An additional 10 patients (15.5\%) showed persistent, activity-limiting shoulder pain that did not permit a return to previous military activities (Table 4).

All variables assessed from univariate analysis were not found to be significantly associated with recurrent instability or persistent pain. These variables included sex (OR, 2.29; 95\% CI, 0.09-58.54), age as a continuous variable (OR, 0.9; 95\% CI, 0.77-1.05), insidious onset (OR, 1.45; 95\% CI, 0.36-5.82), predominantly pain symptoms (OR, 1.62; 95\% CI, 0.42-6.27), intraoperative positioning (beach-chair positioning vs lateral decubitus) (OR, 0.12; 95\% CI, 0.01-2.47), low anchor position (between 5- and 7-o'clock positions) (OR, $0.35 ; 95 \%$ CI, 0.05-2.29), number of anchors used (mean, 3.2) (OR, 0.44; 95\% CI, 0.02-11.18), and use of 3 or fewer suture anchors (OR, 1.9; 95\% CI, 0.3610.07) $(P>.05)$ (Table 2).

\section{Discussion}

The findings of this study indicate that primary arthroscopic posterior labral repair provides a reliable rate of glenohumeral stability with improved pain, a low rate of revision surgery $(1.5 \%)$, and a high rate of return to previous military duties $(83 \%)$ at short- to mid-term follow-up. Our study contributes to the currently limited body of literature on outcomes of isolated, primary arthroscopic posterior labral repair in an active military population.

Our results show that the rate of return to duty slightly lags behind the rates of return to sport in studies of athletes. A recent systematic review by Moeller et al. ${ }^{17}$ revealed an $89 \%$ to $96 \%$ rate of return to sport in athletes after undergoing posterior stabilization surgery at a mean follow-up of 37.6 months. In the largest prospective study to date on arthroscopic posterior stabilization in athletes, Bradley et al. ${ }^{2}$ showed a $93.5 \%$ success rate in return to play after posterior stabilization in 200 
shoulders (183 athletes), concluding that arthroscopic labral repair was an effective and reliable treatment for symptomatic unidirectional posterior instability. Moreover, Arner el al. ${ }^{4}$ reported a $93 \%$ return-to-sport rate in a group of 56 American football athletes, with 2 failures $(3.5 \%)$ due to symptom recurrence. However, it is important to realize that the return of athletes to sport does not mean that they are returning to their previous level of competition. Bradley et al. ${ }^{2}$ reported a $64 \%$ rate of return to the previous level of competition despite an overall return-to-sport rate of $90 \%$.

The rate of revision surgery in our study parallels that of other studies within both military and athletic populations. At an average follow-up of 3.04 years, only 1 patient $(2 \%)$ required revision because of recurrent posterior shoulder instability. Similarly, Bottoni et al. ${ }^{5}$ retrospectively compared 30 military patients with posterior instability who underwent open versus arthroscopic repair and found that recurrence rates were similar in both groups, with each group having failure in 1 patient. Savoie et al. ${ }^{10}$ reported similar findings among athletes, with a $97 \%$ stability rate in 92 athletes who underwent arthroscopic posterior capsulolabral repair.

Despite the low rate of revision surgery in our study, $15.5 \%$ of patients had persistent pain, resulting in the inability to return to previous military activity. One reason for this finding could be the inability to modify activities, such as firing rifles, carrying heavy loads for extended periods, participating in combative training, or participating in other high-impact activity, placing patients at increased risk of persistent pain or recurrence. ${ }^{18}$ The rate of persistent pain in this study is similar to that in previous studies on athletes who also are unlikely to be able to modify activity within their sport. A systematic review and meta-analysis by Delong et al. ${ }^{12}$ revealed a rate of persistent pain of $17.5 \%$ among athletes (103 of 748 shoulders). Another possible cause of persistent pain could be unrecognized underlying shoulder hyperlaxity. Bradley et al. ${ }^{2}$ noted that $62.5 \%$ of athletes with failure their primary arthroscopic posterior labral repair possessed signs of inferior instability or multidirectional instability at the time of reoperation. Of the 10 patients $(15.5 \%)$ with persistent pain in our study, none opted to undergo a reoperation and all decided to proceed with the Medical Board Evaluation process. Because of the retrospective nature of the study, it is difficult to ascertain whether these cases are considered true clinical failures (i.e., pain only) versus surgical failures (i.e., patients would benefit from revision surgery). The rate of failure to return to military duty may be confounded by other factors (i.e., comorbidities and secondary gain) and may not solely result from activity-limiting shoulder dysfunction.

The mechanism of injury resulting in posterior instability has been described to stem from a single traumatic episode rather than repetitive microtrauma. ${ }^{19}$ Symptoms frequently developed in our patient population not only from movements such as push-ups and bench press but also from overhead lifting, combative injuries, falls on outstretched hands, blast mechanisms, and tactical vehicle accidents. Thus, the spectrum of mechanisms of injury is likely broader than previously described and not limited to a narrow spectrum of patient presentations. As a result, clinicians should be attentive to these multiple causes of posterior shoulder instability in the military population, with posterior instability tests being a part of every thorough shoulder physical examination. In our study, high sensitivities of the posterior load-and-shift $(98 \%)$, jerk $(95 \%)$, and Kim $(91 \%)$ tests were achieved and confirmed intraoperatively. The sensitivities found within the literature were $73 \%$ to $90 \%$ and $80 \%$ for the jerk and Kim tests, respectively, whereas the posterior load-and-shift test has not yet been evaluated for its diagnostic accuracy. ${ }^{20}$

Male sex, age, insidious onset, predominantly pain symptoms preoperatively, intraoperative positioning, low anchor position, and use of 3 or fewer suture anchors were not significantly associated with recurrent instability or adverse clinical outcomes. However, it must be mentioned that our study is likely underpowered because of the low number of subjects, making a type II error more likely. Given the natural paucity of patients with posterior labral tears of the shoulder, a power analysis to determine the sample size was not performed at the start of the study. As a result, we cannot make true determinations based on our data alone, but we can make comparisons with existing literature. Regarding intraoperative patient positioning, Moeller et al. ${ }^{17}$ performed a systematic review comparing outcomes in patients who underwent arthroscopic posterior stabilization in either the beachchair or lateral decubitus position. They found no differences in patient outcome scores or rates of recurrent instability or return to sport. Regarding suture anchor number, our study found that the number of suture anchors was not significantly associated with recurrent posterior instability or adverse clinical outcomes. This result is in contrast to findings in a study by Boileau et $\mathrm{al}^{21}$ on anterior labral repairs, which concluded that patients receiving 3 or fewer suture anchors were at higher risk of recurrent anterior shoulder instability. Our findings suggest that the number of anchors is not a predictor of clinical failure in posterior instability cases, in contrast to the finding in anterior instability cases. Further research is required to identify modifiable risk factors associated with persistent activitylimiting shoulder pain in this population.

\section{Limitations}

Limitations of this study include its retrospective nature; lack of power because of the small patient sample 
size; involvement of multiple surgeons (7 surgeons), which likely can lead to heterogeneous surgical selection bias; average follow-up period of only 3.04 years; and lack of patient-reported outcome scores. Although our rate of clinical failure due to pain or a decrease in function $(15.5 \%)$ is higher than that reported in other studies, ${ }^{2,4}$ we must also note that a failure to return to military duty may be confounded by comorbidities and secondary gain and may not necessarily be solely due to activity-limiting shoulder dysfunction. In addition, a unique population of military personnel was used in our cohort with specific military requirements that may not be universally applicable. Subgroup analysis was also limited because of the lack of statistical power. A larger sample size may have improved the ability to analyze different risk factors that may predispose patients to posterior labral instability.

\section{Conclusions}

Arthroscopic intervention resulted in a reliable rate of glenohumeral stability with a low rate of surgical revision and a high rate of return to military duty at shortto mid-term follow-up. However, 1 in 6 military service members showed significant, activity-limiting shoulder pain postoperatively that did not permit a return to previous military activities after surgical intervention.

\section{References}

1. Bradley JP, Forsythe B, Mascarenhas R. Arthroscopic management of posterior shoulder instability: Diagnosis, indications, and technique. Clin Sports Med 2008;27: 649-670.

2. Bradley JP, McClincy MP, Arner JW, Tejwani SG. Arthroscopic capsulolabral reconstruction for posterior instability of the shoulder: A prospective study of 200 shoulders. Am J Sports Med 2013;41:2005-2014.

3. Antosh IJ, Tokish JM, Owens BD. Posterior shoulder instability. Sports Health 2016;8:520-526.

4. Arner JW, McClincy MP, Bradley JP. Arthroscopic stabilization of posterior shoulder instability is successful in American football players. Arthroscopy 2015;31: 1466-1471.

5. Bottoni CR, Franks BR, Moore JH, DeBerardino TM, Taylor DC, Arciero RA. Operative stabilization of posterior shoulder instability. Am J Sports Med 2005;33:996-1002.

6. Chang ES, Greco NJ, McClincy MP, Bradley JP. Posterior shoulder instability in overhead athletes. Orthop Clin North Am 2016;47:179-187.
7. Chauhan A, Mosier B, Kelly B, Akhavan S, Frank DA. Posterior shoulder instability in athletes. JBJS Rev 2015;3.

8. McClincy MP, Arner JW, Bradley JP. Posterior shoulder instability in throwing athletes: Case-matched comparison of throwers and non-throwers. Arthroscopy 2015;31: 1041-1051.

9. Mauro CS, McClincy MP, Bradley JP. Effect of glenoid version and width on outcomes of arthroscopic posterior shoulder stabilization. Am J Sports Med 2016;44: 941-947.

10. Savoie FH III, Holt MS, Field LD, Ramsey JR. Arthroscopic management of posterior instability: Evolution of technique and results. Arthroscopy 2008;24:389-396.

11. Owens BD, Duffey ML, Nelson BJ, DeBerardino TM, Taylor DC, Mountcastle SB. The incidence and characteristics of shoulder instability at the United States Military Academy. Am J Sports Med 2007;35:1168-1173.

12. DeLong JM, Jiang K, Bradley JP. Posterior instability of the shoulder: A systematic review and meta-analysis of clinical outcomes. Am J Sports Med 2015;43:1805-1817.

13. Dickens JF, Kilcoyne KG, Haniuk E, Owens BD. Combined lesions of the glenoid labrum. Phys Sportsmed 2012;40:102-108.

14. Song DJ, Cook JB, Krul KP, et al. High frequency of posterior and combined shoulder instability in young active patients. J Shoulder Elbow Surg 2015;24:186-190.

15. Kim SH, Park JS, Jeong WK, Shin SK. The Kim test: A novel test for posteroinferior labral lesion of the shoulder-A comparison to the jerk test. Am J Sports Med 2005;33:1188-1192.

16. Owens BD, Duffey ML, Deberardino TM, Cameron KL. Physical examination findings in young athletes correlate with history of shoulder instability. Orthopedics 2011;34: 460.

17. Moeller EA, Houck DA, McCarty EC, et al. Outcomes of arthroscopic posterior shoulder stabilization in the beachchair versus lateral decubitus position: A systematic review. Orthop J Sports Med 2019;7:2325967118822452.

18. Cho JH, Chung NS, Song HK, Lee DH. Recurrent posterior shoulder instability after rifle shooting. Orthopedics 2012;35:e1677-e1679.

19. Robinson CM, Aderinto J. Recurrent posterior shoulder instability. J Bone Joint Surg Am 2005;87:883-892.

20. Hippensteel KJ, Brophy R, Smith MV, Wright RW. Comprehensive review of provocative and instability physical examination tests of the shoulder. J Am Acad Orthop Surg 2019;27:395-404.

21. Boileau P, Villalba M, Hery JY, Balg F, Ahrens P, Neyton L. Risk factors for recurrence of shoulder instability after arthroscopic Bankart repair. J Bone Joint Surg Am 2006;88:1755-1763. 